\title{
INDUCTIVE DIMENSION AND INVERSE SEQUENCES OF COMPACT SPACES
}

\author{
M. G. CHARALAMBOUS
}

\begin{abstract}
We construct an inverse sequence of compact Hausdorff spaces each of which has inductive dimension one while the limit space of the sequence has large inductive dimension two.
\end{abstract}

1. Introduction. The inverse limit theorem for covering dimension, dim, states that if $X$ is the limit space of an inverse system $\left\{X_{\alpha}, f_{\alpha \beta}\right\}$ where each $X_{\alpha}$ is compact with $\operatorname{dim} X_{\alpha} \leqslant n$, then $\operatorname{dim} X \leqslant n$. The inverse limit theorem for $\operatorname{dim}$ also holds for inverse sequences of perfectly normal spaces [1], but it fails for inverse sequences of Lindelöf spaces [2]. In this paper, we investigate the inverse limit theorem for large inductive dimension, Ind. It is easy to see that this theorem fails for arbitrary inverse systems of compact spaces on account of the fact that every compact space $X$ with $\operatorname{dim} X \leqslant n$ is the limit space of an inverse system of compact metric spaces $X_{\alpha}$ with $\operatorname{dim} X_{\alpha} \leqslant n$ : it suffices to consider a compact space $X$ with $\operatorname{dim} X<$ Ind $X$. Our aim is to show that the inverse limit theorem fails even for inverse sequences of compact spaces. We construct an inverse sequence $\left\{X_{n}, \pi_{n m}\right\}$ of compact spaces $X_{n}$ with Ind $X_{n}=1$ whose limit space $X$ satisfies Ind $X=2$. Our construction was suggested by a construction of Fedorčuk in [4].

All results and terminology pertaining to inverse limits and dimension that are referred to in this paper can be found in both [3] and [5].

2. The construction. Let $I$ denote the closed interval $[0,1], J$ the open interval $(0,1)$ and $N$ the set of positive integers. Let $A_{1}, A_{2}, \ldots$ be a partition of $J$ into disjoint dense sets. Let $\left\{F_{\alpha}: \alpha<c\right\}$ be the collection of all perfect and, therefore, uncountable sets of $I$, where $c$ is the cardinality of the continuum, and for each $\alpha<c$ choose a double-sided point $x_{\alpha}$ in $F_{\alpha}$ so that $x_{\alpha} \neq x_{\beta}$ for $\alpha \neq \beta$. For each $x$ in $J$ choose an increasing sequence $\left\{x_{n}^{-}\right\}$and a decreasing sequence $\left\{x_{n}^{+}\right\}$, both converging to $x$ and such that, if $x=x_{\alpha}$ for some $\alpha<c$, then $x_{n}^{-}$and $x_{n}^{+}$are in $F_{\alpha}$ for each $n$ in $N$.

For each $x$ in $J$, we define a continuous function $f=f_{x}: I-\{x\} \rightarrow I$ as follows. We set $f(0)=0, f(1)=1$ and $f\left(x_{n}^{-}\right)=f\left(x_{n}^{+}\right)=q_{n}$, where $Q=\left\{q_{n}: n \in N\right\}$ is a dense set of $J$. To the intervals $\left[0, x_{1}^{-}\right],\left[x_{1}^{-}, x_{2}^{-}\right], \ldots,\left[x_{1}^{+}, 1\right],\left[x_{2}^{+}, x_{1}^{+}\right], \ldots, f$ is extended in the obvious homeomorphic fashion. It can readily be seen that for every $y$ in $J, f^{-1}(y)$ consists of an increasing together with a decreasing sequence, both converging to $x$, and if $A$ is a countable dense subset of $J$, then so is $f^{-1}(A)$.

Received by the editors November 23, 1979 and, in revised form, March 10, 1980.

1980 Mathematics Subject Classification. Primary 54F45, 54G20.

Key words and phrases. Inverse sequence, covering dimension, inductive dimension. 
Now, if $\left\{B_{1}, B_{2}, \ldots\right\}$ and $\left\{C_{1}, C_{2}, \ldots\right\}$ are collections of mutually disjoint countable dense sets of $J$, there is a homeomorphism $g: J \rightarrow J$ with $g\left(B_{1}\right)=C_{1}$, $g\left(B_{2}\right)=C_{2}, \ldots$ It follows that we may assume that there are countable dense sets $Q_{m}$ of $J, m \in N$, such that $f^{-1}\left(Q_{m}\right)$ is a dense subset of $A_{m}$.

For $n=1,2, \ldots, \infty$, we let

$$
Y_{n}=\bigcup_{i=1}^{n} A_{i} \times I \times\{i\} \cup I \times\{0\} \times\{i\},
$$

$X_{n}$ is the set obtained from $Y_{n}$ by identifying two points $\left(x_{1}, y_{1}, i\right),\left(x_{2}, y_{2}, j\right)$ if and only if $x_{1}=x_{2}$ and $y_{1}=y_{2}=0$. Thus the point $(x, 0, i)$ of $X_{n}$ may be more simply written as $x$. The projection $\pi_{n m}: X_{m} \rightarrow X_{n}, m \geqslant n$, is defined by $\pi_{n m}(x, y, i)=$ $(x, y, i)$ for $i<n$ and $\pi_{n m}(x, y, i)=x$ for $i>n$. A function $\pi=\pi_{n}: X_{n} \rightarrow I$ is defined by $\pi(x, y, i)=x$. Finally, we define the topology of $X_{n}$ by defining the local base of open neighbourhoods at each point of $X_{n}$. At a point $x$ with $x \notin \cup_{i=1}^{n} A_{i}$, we take as base the collection of all sets of the form $\pi^{-1}(U)$, where $U$ is an open neighbourhood of $x$ in $I$. At a point $(x, y, i)$ where $x \in A_{i}$ and $i<n$, we take as base the collection of all sets of the form

$$
O(x, U, V)=\{x\} \times V \times\{i\} \cup \pi^{-1}\left(U \cap f_{x}^{-1}(V)\right)
$$

where $U, V$ are open neighbourhoods of $x, y$, respectively, in $I$.

It is readily seen that $\pi: X_{n} \rightarrow I$ and $\pi_{n m}: X_{m} \rightarrow X_{n}, n=1,2, \ldots, \infty$, are continuous and $\left\{X_{n}, \pi_{n m}, n, m \in N\right\}$ is an inverse sequence with limit space $X=X_{\infty}$.

Claim 1. For $n=1,2, \ldots, \infty, X_{n}$ is compact and Hausdorff.

Proof. That $X_{n}$ is Hausdorff is straightforward. To see that $X_{n}$ is compact, suppose $\left\{O\left(x, U_{\alpha}, V_{\alpha}\right): \alpha \in \Lambda\right\}$ is an open cover of $\{x\} \times I \times\{i\}, x \in A_{i}$ and $i<n$, which is homeomorphic with $I$. Then for some $\alpha_{1}, \ldots, \alpha_{k}$ in $\Lambda, I=V_{\alpha_{1}}$ $\cup \cdots \cup V_{\alpha_{k}}$. Letting $U=U_{\alpha_{1}} \cap \cdots \cap U_{\alpha_{k}}$, we see that $O\left(x, U, V_{\alpha_{k}}\right)$ $\cup \cdots \cup O\left(x, U, V_{\alpha_{k}}\right)=\pi^{-1}(U)$ contains $\{x\} \times I \times\{i\}$. Thus for every $x$ in $I$ and every open cover $\left\{G_{\alpha}\right\}$ of $\pi^{-1}(x)$, there is an open neighbourhood $U$ of $x$ in $I$ such that $\pi^{-1}(U)$ is covered by a finite number of $G_{\alpha}$ 's. This readily implies that $X_{n}$ is compact.

Claim 2. For each $n$ in $N$, Ind $X_{n}=1$.

Proof. Since $\pi^{-1}(x)$ is homeomorphic with $I$ for each $x$ in $A_{n}$, then Ind $X_{n}>1$. It suffices, therefore, to show that Ind $X_{n}<1$. For a point $(x, 0, i)$ of $X_{n}$ with $x \notin \cup_{i=1}^{n} A_{i}$, we can choose inside any given neighbourhood, a neighbourhood of the form $\pi^{-1}(U)$ where $U=(a, b)$ or $[0, b)$ or $(a, 1]$ for some $a, b$ in $A_{n+1}$. Clearly $B\left(\pi^{-1}(U)\right)$, where $B$ denotes boundary consists, of one or both of $a, b$. For a point $(x, y, i)$ with $x$ in $A_{i}$ and $i \leqslant n$, inside any neighbourhood we can choose a neighbourhood $O(x, U, V)$ with $V=(a, b)$ or $[0, a)$ or $(b, 1]$ where $a, b$ are points of $Q_{n+1}$ and $U$ is an open interval with end points chosen from the countable subset $f_{x}^{-1}\{a, b\}$ of $A_{n+1}$. It can be verified that $B(O(x, U, V))$ is a subset of $\{x\} \times\{a, b\} \times\{i\} \cup f_{x}^{-1}\{a, b\}$. We conclude that ind $X_{n}<1$ and hence Ind $X_{n}$ $<1$.

Claim 3. Ind $X=2$. 
Proof. As in the proof of Claim 2, every point of $X$ has a base of open sets whose boundary consists of a countable number of copies of $I$ together with at most two points. Hence Ind $X \leqslant 2$ and it remains to show Ind $X>2$. Let $G, H$ be disjoint nonempty open subsets of $X$ such that $X=G \cup H \cup F$, where $F=\bar{G} \cap$ $\bar{H}$. It suffices to show that $F$ contains a copy of $I$. Suppose first that $\pi(F)$ is a perfect subset of $I$. Then for some $\alpha<c, \pi(F)=F_{\alpha}$, and it can be readily verified that $O(x, U, V)$, where $x=x_{\alpha}, U$ is an open neighbourhood of $x$ in $I$ and $V$ is any nonempty open subset of $I$, contains infinitely many of $\pi^{-1}\left(x_{n}^{-}\right)$and $\pi^{-1}\left(x_{n}^{+}\right)$, $n \in N$. Since $x_{n}^{-}, x_{n}^{+}$were chosen to be points of $\pi(F), O(x, U, V)$ contains infinitely many points of $F$. It follows that $\pi^{-1}(x)$, a copy of $I$, is contained in $F$. Finally, suppose $\pi(F)$ is not perfect, i.e., $\pi(F)$ contains an isolated point, say, $x$. Since $\pi^{-1}(y)$ is connected for each $y$ in $I$, it follows that $I=\pi^{*}(G) \cup \pi^{*}(H) \cup$ $\pi(F)$, where for a subset $A$ of $X, \pi^{*}(A)=\left\{y \in I: \pi^{-1}(y) \subset A\right\}$. Let $U=(a, b)$ be an open neighbourhood of $x$ such that $U \cap \pi(F)=\{x\}$. We must have $U \cap$ $\pi^{*}(G) \neq \varnothing$ and $U \cap \pi^{*}(H) \neq \varnothing$; otherwise $\pi^{-1}(U) \cap G$ or $\pi^{-1}(U) \cap H$ are subsets of $\pi^{-1}(x)$. Hence, without loss of generality, $(a, x) \subset \pi^{*}(G)$ and $(x, b) \subset$ $\pi^{*}(H)$. For any given nonempty open set $V$ of $I$, choose $n$ in $N$ so that $f\left(x_{n}^{-}\right)=f\left(x_{n}^{+}\right)$is in $V$ and $a<x_{n}^{-}<x_{n}^{+}<b$. Then $O(x, U, V)$ contains both $\pi^{-1}\left(x_{n}^{-}\right)$, a subset of $G$, and $\pi^{-1}\left(x_{n}^{+}\right)$, a subset of $H$. It follows that every point of $\pi^{-1}(x)$ is a limit point of both $G$ and $H$ and hence $\pi^{-1}(x) \subset F$. This concludes the proof since $\pi^{-1}(x)$ is homeomorphic with $I$.

We note in conclusion that for $n=1,2, \ldots, \infty, X_{n}$ is both separable and first countable. Also, since Ind $X_{n}=1$, for each $n$ in $N, \operatorname{dim} X_{n}=1$ and, by the inverse limit theorem for $\operatorname{dim}, \operatorname{dim} X=1$.

\section{REFERENCES}

1. M. G. Charalambous, The dimension of inverse limits, Proc. Amer. Math. Soc. 58 (1976), 289-295.

2. An example concerning inverse limit sequences of normal spaces, Proc. Amer. Math. Soc. 78 (1980), 605-607.

3. R. Engelking, Dimension theory, Polish Scientific Publishers, Warsaw, 1978.

4. V. V. Fedorcuk, Compact spaces without intermediate dimensions, Soviet Math. Dokl. 14 (1973), 1808-1811.

5. A. R. Pears, Dimension theory of general spaces, Cambridge University Press, Cambridge, 1975.

Department of Mathematics, University of NaIrobi, NaIrobi, KenYa 